\title{
MEMORIA Y OLVIDO: PARTICIPACIÓN DE ANTONIA MORENO DURANTE LA CAMPAÑA DE LA BREÑA. ANDES CENTRALES (PERÚ), 1881-1883
}

Luz Estefany Ramos-Dolorier*

\section{Bibliografía anotada}

\section{Presentación}

Este trabajo intenta dar a conocer la historia de las mujeres durante el desarrollo del conflicto bélico Perú-Chile (1879-1883), particularmente la figura de Antonia Moreno de Cáceres, mujer de clase alta. En dicha oportunidad se publicará tal volumen en el que está presente la bibliografía anotada.

\section{Bibliografía y fuentes}

$\mathrm{Al}$ igual que cualquier otra bibliografía, la que presentamos dará mayor acercamiento a los/las investigadoras interesadas en insertarse en la historia de las mujeres en el Perú; particularmente, analizar el accionar de las mujeres durante la guerra con Chile. El presente personaje por investigar y esbozar una biografía detallada y ampliada es el de Antonia Moreno, conocida como la “mamacha” y cónyuge del Mariscal Andrés Avelino Cáceres.

Si bien existen investigaciones referentes a la historia de las mujeres en dicho contexto histórico, consideramos importante continuar contribuyendo con la visibilización y reivindicación de las mujeres como sujetos históricos. Cabe mencionar las limitadas fuentes y bibliografía referente al tema en desarrollo, sin embargo, discurrimos significativamente la elaboración de dicho trabajo para un mayor rastreo de la producción historiográfica actualizada, puesto que las últimas referencias fueron de los nuevos análisis y aportes en la disciplina literaria y artística elaborada por Ruth Solarte, Isabelle Tauzin y Nanda Leonardini.

\footnotetext{
*Universidad Nacional Federico Villarreal. Lima, Perú. E-mail: dolorier_11@hotmail.com.

(D) orcid.org/0000-0002-1638-1552.
} 
Como advertencia se debe considerar que:

a. La bibliografía plasmada posee como referencia los escritos de Sara Beatriz Guardia y Maritza Villavicencio, investigadoras peruanas que se han dedicado a la producción historiográfica de las mujeres, buscando una reivindicación de dicho papel.

\section{Libros, tesis y folletos}

\section{- Por contexto histórico}

Basadre, J. (2014). Historia de la República del Perú 1822-1993. Lima, Perú: Colección El Comercio.

Este libro analiza en diversas perspectivas los factores que generaron la guerra, cómo se fue desarrollando en distintos puntos del país y de las severas consecuencias que conllevó, utilizando fuentes de primera mano y diversos testimonios. Basadre, historiador tacneño, apreciado especialista en la época republicana peruana, sus trabajos son considerados como referencia obligatoria para todo aquel investigador que desee adentrarse en dicho siglo. Tal texto forma parte de toda una colección realizada por El Comercio de 15 tomos, que abarcan desde el periodo de 1822 al 2000. Sin lugar a duda, una obra importante en la producción historiográfica del siglo XX.

Contreras, C. y Cueto, M. (2004). Historia del Perú Contemporáneo (5ta edición). Lima, Perú: Instituto de Estudios Peruanos.

Este libro presenta una interesante síntesis de la historia republicana del Perú, colocando a debate y aplicación de grandes proyectos de reforma política ocurridos en los últimos 200 años. Además, desarrollan tal investigación con más información como: datos estadísticos, cuadros comparativos y datos cuantitativos acerca de todo lo relacionado antes, durante y después de la guerra Perú-Chile. Los autores, historiadores reconocidos que ejercen la docencia universitaria, actualmente especialistas en historia económica y salud en el Perú, respectivamente. Este estudio se enmarca como un texto de lectura referencial para la formación de los historiadores(as) de diversas especialidades. 
Kauffman, D.F., Porras, B.R. y Vargas U.R. (1986). Historia General de Los Peruanos. Lima, Perú: PEISA.

Esta colección presenta los resultados de exhaustivas investigaciones realizadas por tales autores, particularmente los aportes de Rubén Ugarte, acerca de los diversos acontecimientos históricos. Se destaca el buen uso de las fuentes documentales que en tales años recién se exploraba, sin dejar de mencionar la invisibilidad hacia el género femenino en el accionar de acontecimientos históricos particularmente de los años 1879-1883. Tal texto fue escrito por el destacado historiador, quien fue rector de la Pontificia Universidad Católica del Perú, Rubén Vargas hasta el Volumen X. El presente libro ha sido publicado por la reconocida editorial emblemática peruana Milla Batres en el año 1966.

\section{- Por las referencias en torno a la historia de las mujeres}

Scott, J. (2008). Género e Historia. Ciudad de México, México: Fondo de Cultura Económica y Universidad Autónoma de la Ciudad de México.

Este libro categoriza de manera clara y precisa la construcción teórica del género, las desigualdades entre los sexos y la inclusión de las mujeres como sujetos históricos. Expone el género como categoría de análisis y el cómo los avances de las investigaciones feministas permitieron una mayor consolidación de los movimientos feministas surgidos en Norteamérica. Joan Scott, distinguida historiadora pionera en los estudios de género estadounidenses, reconocida por sus diversas investigaciones y aportes que ha brindado para tal campo. Tal texto sirve como referente a todo investigador/ra que se adentre a tal temática.

Perrot, M. (2008). Mi historia de las mujeres. Madrid, España: Fondo de Cultura Económica.

Este libro permite comprender la historia de las mujeres al expandir sus perspectivas espaciales, religiosas y culturales. Analiza el proceso de desarrollo de la diferencia entre los sexos, la jerarquización del poder y la configuración de sus identidades. Explora la cotidianidad de las mujeres en sus diversos ámbitos: las campesinas, las obreras, costureras, enfermeras, domésticas, etc. y, de su participación en diversos acontecimientos históricos. Michel Perrot, historiadora feminista pionera en los estudios de la historia de las mujeres en Francia, por sus textos en colaboración con Georges Duby, entre otros. La autora llevó a cabo la presente investigación con el auspicio del Fondo de Cultura Económica de Buenos Aires. 
Ramos, C. (1992). Género e Historia. La historiografía sobre la mujer. Ciudad de México, México: Universidad Autónoma Metropolitana.

Este libro analiza la compleja evolución en el campo de los estudios de las mujeres en los últimos tiempos, contando con principales teóricos e incluyendo ensayos de destacadas exponentes internacionales. Reflexiona sobre los problemas que se puede plantear para la historiografía mexicana relacionada a la historia de las mujeres, y como la categoría género puede modificar la perspectiva sobre dichos problemas. Claro está que tal escrito sirve como un modelo a seguir; teniendo en cuenta del cómo se puede insertar esta categoría en los problemas sociales de los diversos países sudamericanos, relacionados directamente al género e historia de las mujeres. Carmen Ramos, reconocida historiadora mexicana, fundadora de la cátedra Metodología de género e Historia de la mujer en México en la Universidad Nacional Autónoma de México.

Guardia, S. y Andreo, J. (2002). Historia de las Mujeres en América Latina. Lima, Perú: Centro de Estudios la Mujer en la Historia de América Latina.

El presente libro presenta las investigaciones de la historia de la mujer peruana. Exponen el reconocimiento de las experiencias femeninas, significando un aporte importante para la historiografía del siglo XX. El esfuerzo por reconstruir dicho pasado femenino debe entenderse fundamentalmente como un marco conceptual que va permitiendo descubrir ese otro lado de la historia. Los autores llevaron a cabo dicha investigación bajo el auspicio del Centro de Estudios de la Mujer en la Historia de América Latina. Dicho libro se enmarca en los aportes historiográficos trascendentales en la historia de las mujeres en el Perú.

Guardia, S. (2002). Mujeres peruanas el otro lado de la historia. Lima, Perú: Minerva- CEMHAL.

La investigadora Sara Beatriz Guardia, pionera en los estudios de la mujer en el Perú, expone sus conclusiones acerca de la importancia de rescatar del olvido la narración de las mujeres peruanas en los distintos periodos históricos, desde el Antiguo Perú hasta la inserción de las mujeres en la política peruana. El análisis de la mujer como sujeto histórico resulta un creciente avance de la historia social, surgiendo así una serie de nuevas voces a individuos que estuvieron olvidados por la historia. Este trabajo es considerado una de las publicaciones pioneras en los estudios femeninos peruanos. Sara Beatriz Guardia, investigadora y escritora reconocida, fundadora del Centro de Estudios de la Mujer en la Historia de América Latina y autora de diversos libros. 
Meza, C. y Hampe, T. (2007). La Mujer en la Historia del Perú (siglo XV al XX). Lima, Perú: Fondo Editorial del Congreso del Perú.

Este libro reúne los resultados de las diversas investigaciones relacionadas a la historia de las mujeres peruanas. Se analiza explícitamente con el buen manejo de las fuentes documentales inéditas como: Colección del Archivo General de la Nación (colonia y república), Archivo Histórico de la Biblioteca Nacional del Perú, Colección Hemerográfico del Instituto de Riva Agüero, etc., acerca del rol importante que desempeñó la mujer en los diversos periodos de la historia. Entre ellas, destacamos a los compiladores Teodoro Hampe y Margarita Zegarra, historiadores importantes especialistas en el periodo colonial y republicano, respectivamente; y que sus aportes han sido de suma importancia para la producción historiográfica contemporánea en los estudios de la mujer.

Denegri, F. (1996). El abanico y la cigarrera. La primera generación de mujeres en el Perú. Lima, Perú: Instituto de Estudios Peruanos (IEP).

Francesa Denegrí, destacada literata de la Pontificia Universidad Católica del Perú, especialista en los estudios de las mujeres en el siglo XIX, argumenta que las veladas literarias organizadas por Clorinda Matto de Turner y Mercedes Cabello, generaron importantes aportes en la escritura peruana, permitiendo abrir espacios para que las mujeres fueran escuchadas. El discurso de tales veladas estaba íntimamente ligada a las políticas feministas construidas por mujeres que se asocian para reclamar derechos para ellas. Se visibilizó una rebeldía femenina a través de la escritura colocándose seudónimos, rompiendo con los estereotipos de los conservadores del siglo XIX. Dicha autora llevó a cabo esta investigación bajo los auspicios del Centro de la mujer peruana Flora Tristán y el Instituto de Estudios Peruanos.

Villavicencio, M. (1992). Del silencio a la palabra. Mujeres peruanas en el siglo XIX-XX. Lima, Perú: Editorial del centro de la mujer peruana Flora Tristán.

Este libro presenta los resultados de una investigación sobre el origen del movimiento de mujeres a través de la historia peruana. Su división ha sido abordada desde la colonia hasta el surgimiento del trabajo femenino durante la etapa de modernización del Perú. Cabe mencionar que dicho trabajo posee una visión reivindicativa de la mujer como protagonista y a la vez, enriquecedora del estudio de un grupo de mujeres y que, en su momento, fue muy poco abordado desde una nueva perspectiva. Maritza Villavicencio, historiadora y autora del libro "Mujer, poder y alimentación en el Antiguo Perú" (2017), y especialista en los estudios prehispánicos en el Perú. 
Bejarano, G.E. (2011). Elvira García y García (1892-1951): mujer y educadora dentro de los procesos modernizadores de la educación de la infancia en el Perú. Lima, Perú: Universidad Nacional Mayor de San Marcos (UNMSM).

El investigador de la Universidad Nacional Mayor San Marcos, Emilio Bejarano, expone en su tesis el importante papel de Elvira García y García en la educación femenina a inicios de la república peruana. El enriquecedor trabajo brinda mayores aportes en sus primeros capítulos referentes al contexto histórico, las deficiencias de las políticas educativas en la república y el rol definido hacia las mujeres en el hogar desde el siglo XIX al XX. La presente tesis ha sido desarrollada y estructurada para optar el grado de licenciatura de la especialidad de historia en la Universidad Nacional Mayor de San Marcos.

Zegarra, M. (2011). María Jesús Alvarado Rivera: la construcción de una intelectual feminista en Lima, 1878-1915. Lima, Perú: UNMSM.

Este libro presenta los resultados de una exhaustiva investigación sobre la importancia del papel de María Jesús Alvarado, intelectual peruana que brindó grandes aportes en su época. Considerada como una de las pioneras del feminismo intelectual peruano que propuso un discurso anti-dogmático, denunciando que la Iglesia y la sociedad limeña dominaban a la mujer para cumplir con su rol de ama del hogar. El presente trabajo brinda mayores aportes para la construcción de una historia feminista peruana; sin embargo, para dicha investigación tiene mayor preponderancia en el análisis realizado en los primeros capítulos referentes: al contexto histórico y del surgimiento de una república temprana domina por la alta élite. Margarita Zegarra es historiadora y autora de diversos escritos importantes, destacándose la dirección a su cargo del Centro de Documentación sobre la Mujer (CENDOC-MUJER).

Mendoza, G. (1987). Antonia Moreno de Cáceres. Lima, Perú: Colección El Comercio.

Este ensayo brinda un análisis resumido de la figura de Antonia Moreno durante su accionar en la campaña de la Breña. Argumenta de manera inteligible y concreta las acciones de Moreno desde su condición como madre, patriota y mujer en dicha campaña. Tal trabajo visibiliza la imagen histórica femenina de los personajes que participaron en la guerra Perú-Chile, particularmente la figura de Antonia Moreno en la historia. Recién cuando la producción historiográfica femenina logró una mayor importancia en el Perú, ello incentivó que los diversos diarios realizaran concursos ensayísticos de este tipo. Sobre la autora, se desconocen sus datos relevantes puesto que en todo el documento no se menciona una breve hoja de vida. 


\section{Artículos en revistas (autores relacionados al contexto y luego específicos en orden alfabético)}

\section{- Por contexto histórico}

Rosario, E. (2008). Por el devenir de un gran paradigma nacional: un balance historiográfico a la guerra del pacífico. Revista Investigaciones Social, XII (20), 301-334.

El investigador de la Universidad Nacional Mayor de San Marcos, Emilio Rosario, presenta en su trabajo un estado de la cuestión referente a la Guerra del Pacífico. Tal trabajo utiliza los estudios tradicionalistas que marcaron líneas historiográficas como: Manuel González Prada, José Carlos Mariátegui, Luis Alberto Sánchez, entre otros. Resalta el surgimiento de una historiografía de resistencia que lucha a ser absorbida por la tradición; difundiéndose libros que argumentan otra óptica de la guerra, gracias a las fuentes documentales inéditas que contribuyeron con dicha perspectiva.

\section{- Por las referencias en torno a la historia de las mujeres peruanas}

Kogan, L. (1996). Estudios sobre relaciones de género en los sectores medios y altos de Lima. P. Ruiz-Bravo. y L. Kogan. (Eds.), Detrás de la puerta: hombres y mujeres en el Perú de hoy (pp 192-208). Lima, Perú: Pontificia Universidad Católica del Perú.

El presente trabajo presenta una bibliografía anotada referente a los sistemas de género en las familias del sector alto. Argumenta que dicho sistema de género que se construye es el de la femineidad y la masculinidad como dos esferas, fomentando la incomunicación entre géneros que han sido definidos como constructores polares. Referencia las posturas de Maruja Barrig, Norma Fuller, entre otras. Complementa tal trabajo con los diversos datos cuantitativos y cualitativos, que permiten una mayor amplitud en la organización del trabajo. Liuba Kogan, antropóloga e investigadora destacada por la Universidad del Pacífico, ha sido miembro del Comité Editorial de la Revista Latinoamérica de Estudios sobre Cuerpos, Emociones y Sociedad (RELACES), Asociación Latinoamericana de Investigadores en Comunicación (ALAIC) y Latin American Studies Association (LASA).

Van Deusen, N.E. (2002). Voces y silencios: el género en la historia peruana (1977-2002). Revista Histórica, XXVI (1-2). 125-186.

Nancy E. Van Deusen, historiadora de Western Washington University, especialista en la historia colonial de Latinoamérica. Tal trabajo es una bibliografía anotada relacionada a las diversas tendencias, enfoques y las contribuciones referidas a los periodos prehispánico y colonial en la 
historia de las mujeres y al género en los Andes. Expresa que el papel de la mujer en la familia sirvió como un medio viable para influir en las estructuras políticas y económicas. En años posteriores, fue manteniendo dicha estructura con ciertas modificaciones, hasta revolucionar en la época republicana donde su independencia económica y el surgimiento de mujeres en los sectores populares urbanos, permitieron un mayor análisis de estudio. La autora llevó a cabo dicha investigación bajo los auspicios del Centro de Documentación de la Mujer (CENDOCMUJER) y la Pontificia Universidad Católica del Perú (PUCP).

Tauzin, I. (1995). La Narrativa Femenina en el Perú antes de la guerra del Pacífico. Revista Crítica Literaria Latinoamericana, XXI (42), 161-187.

Isabel Tauzin, destacada investigadora francesa de la Université Bordeux Montaige, autora que ha publicado diversos textos en francés y español acerca de la importancia de la escritura femenina peruana. Este libro presenta los resultados del surgimiento de las mujeres escritoras en los inicios del siglo XIX, la influencia del contexto sociocultural, el cambio de rol que cumplió la literatura peruana. Tal texto permite comprender la inserción de la presencia femenina en la literatura peruana que generaría una visibilización de las mujeres en diversos espacios socioculturales. Este estudio se llevó a cabo bajo los auspicios de la Revista de Crítica Literaria Latinoamericana.

\section{- Con relación al tema en específico}

Villavicencio, M. (2013). Acción de las mujeres peruanas durante la guerra con Chile. Revista Debates en sociología, (10), 147-158.

Este artículo presenta las primeras aproximaciones del accionar de las mujeres en las guerras, particularmente el conflicto bélico Perú-Chile. Tal investigación visibiliza a las mujeres como sujetos históricos que pasaron en el anonimato, cuyos actos cotidianos y heroicos hacen posible desde la propia existencia de los ejércitos hasta el punto de garantizar la resistencia y las victorias. Utiliza como fuente importante las memorias y escritos de Antonia Moreno y Elvira García y García, que permitieron contribuir con nuevas e interesantes perspectivas de la guerra. Dicho trabajo deja abierto a un mayor análisis en la investigación, puesto que existe una limitada fuente documental debido a un alto índice de analfabetismo de mujeres pobres en tales años que no permite ir más allá del accionar de las rabonas en la guerra Perú-Chile. 
Rodríguez, D.J. (2009). El bello sexo en guerra: cultura política y género durante la guerra del Pacífico. Lima, PERÚ: Illapa $\mathrm{N}^{\circ} 5$.

Jaime Rodríguez, licenciado en educación y estudios de Maestría en Historia por la Universidad Nacional Mayor de San Marcos, especialista en la Guerra con Chile, ha publicado varios artículos referentes al tema. Expone la importancia de la lectura de los documentos, revistas, periódicos, cartas, informes y memorias de guerra, que permiten obtener una visión alternativa a la historia tradicional. Argumenta que los primeros estudios relacionados a la historia de la guerra Perú-Chile han sido elaborados con la intención de enaltecer al sexo masculino, surgiendo así en los diversos textos escolares el discurso del sexo débil, el de la mujer. El autor llevó a cabo tal investigación bajo el auspicio de Sociedad de Estudios Históricos Coronel Arnaldo Panizo.

Leonardini, H.N. (2014). Presencia femenina durante la Guerra del Pacífico. El caso de las Rabonas. NORBA. Revistas de Arte, XXXIV, 177-195.

En el presente texto, la autora brinda una peculiar perspectiva referente a las rabonas y mujeres de alta clase que participaron en la Guerra del Pacífico, y que lograron pasar a la inmortalidad gracias a la pintura y al grabado. Analizando las pinturas de Alberto Tauro del Pino, Carlos Baca Flor, Carlos Prince, entre otros, argumenta que a través de las diversas obras de arte han permitido conservar la imagen de mujeres de clase alta, en ciertos casos y de mujeres anónimas. En este caso, la figura de Antonia Moreno en la pintura ha sido muy poco estudiada e invisibilizada; generando incluso en un determinado momento dibujos satíricos de los esposos Cáceres Moreno, buscando ridiculizar la figura de estos.

Solarte, G.R. (2018). Desplazamientos y resistencia femenina durante la guerra del Pacífico: las memorias de Antonia Moreno de Cáceres. Revista Decimonónica, 15 (1), 50-66. Recuperado de http://www.decimononica.org/solarte-15-1/.

Este libro postula interesantes propuestas en torno a la memoria "Recuerdos de la Campaña de La Breña" (1974) de Antonia Moreno, como un texto feminista que presenta un sesgo indigenista. Expone que es un texto bélico y de viaje femenino, introduciéndose en la sección de libros de viajeras en este caso de una peruana de clase alta. Tal texto permite dar otra mirada desde la literatura, la memoria de Moreno y del bagaje de información que de este devienen. Ruth Solarte, literata colombiana e investigadora del Instituto Kellogg for International Studies, especialista en los estudios literarios latinoamericanos de género. 


\section{Conclusiones}

Evidentemente la producción historiográfica en los estudios de género y las mujeres en América Latina ha brindado diversas miradas que permiten así, rescatar del olvido a mujeres que contribuyeron en los procesos históricos.

Durante el conflicto bélico entre Perú y Chile (1879-1883), los variados enfrentamientos/ batallas produjeron una fuerte mortandad masculina, exigiendo a las mujeres a subsistir frente a sus escasos recursos económicos. El Perú estuvo azotado por una fuerte crisis financiera durante y después de la guerra, y todo ello repercutió en la dinámica social peruana. Consecuentemente, fueron las mujeres quienes tuvieron que resistir y apoyar hasta la finalización de dicha guerra, con la firma del Tratado de Ancón (1883).

La presente bibliografía anotada recopila las diversas hipótesis sobre la figura de Antonia Moreno de Cáceres, mujer de clase alta y cónyuge de Andrés Avelino Cáceres, quien participó aguerridamente durante la Campaña de La Breña. Igualmente, permite visibilizar los diversos vínculos/redes existentes entre mujeres peruanas de diversas clases para lograr sus objetivos.

Las investigaciones analizadas en el presente trabajo llaman el interés a continuar abordando la imagen de esta mujer, dado que claramente existe una influencia nacionalista manifestado en su fervor patriótico en dicha contienda bélica, y del cómo la población peruana sean mujeres, niños, ancianos, entregaron sus vidas por defender el territorio.

\section{Referencias}

Basadre, J. (2014). Historia de la República del Perú 1822-1993. Lima, Perú: Colección El Comercio.

Bejarano, G.E. (2011). Elvira García y García (1892-1951): mujer y educadora dentro de los procesos modernizadores de la educación de la infancia en el Perú. Lima, Perú: Universidad Nacional Mayor de San Marcos (UNMSM).

Contreras, C. y Cueto, M. (2004). Historia del Perú Contemporáneo. Lima, Perú: Instituto de Estudios Peruanos.

Denegri, F. (1996). El abanico y la cigarrera. La primera generación de mujeres en el Perú. Lima, Perú: Instituto de Estudios Peruanos (IEP).

Guardia, S. (2002). Mujeres peruanas el otro lado de la historia. Lima, Perú: Minerva- CEMHAL.

Guardia, S. y Andreo, J. (2002). Historia de las Mujeres en América Latina. Lima, Perú: Centro de Estudios la Mujer en la Historia de América Latina. 
Kauffman, D.F., Porras, B.R. y Vargas U.R. (1986). Historia General de Los Peruanos. Lima, Perú: PEISA.

Kogan, L. (1996). "Estudios sobre relaciones de género en los sectores medios y altos de Lima”. En P. Ruiz-Bravo. y L. Kogan. (Eds.), Detrás de la puerta: hombres y mujeres en el Perú de hoy (pp 192-208). Lima, Perú: Pontificia Universidad Católica del Perú.

Leonardini, H. N. (2014). Presencia femenina durante la Guerra del Pacífico. El caso de las Rabonas. NORBA. Revistas de Arte, XXXIV, 177-195.

Mendoza, G. (1987). Antonia Moreno de Cáceres. Lima, Perú: Colección El Comercio.

Meza, C. y Hampe, T. (2007). La Mujer en la Historia del Perú (siglo XV al XX). Lima, Perú: Fondo Editorial del Congreso del Perú.

Perrot, M. (2008). Mi historia de las mujeres. Madrid, España: Fondo de Cultura Económica.

Ramos, C. (1992). Género e Historia. La historiografía sobre la mujer. Ciudad de México, México: Universidad Autónoma Metropolitana.

Rodríguez, D. J. (2009). El bello sexo en guerra: cultura política y género durante la guerra del Pacífico. Lima, PERÚ: Illapa $\mathrm{N}^{\circ} 5$.

Rosario, E. (2008). Por el devenir de un gran paradigma nacional: un balance historiográfico a la guerra del pacífico. Revista Investigaciones Social, XII (20), 301-334.

Scott, J. (2008). Género e Historia. Ciudad de México, México: Fondo de Cultura Económica y Universidad Autónoma de la Ciudad de México.

Solarte, G.R. (2018). Desplazamientos y resistencia femenina durante la guerra del Pacífico: las memorias de Antonia Moreno de Cáceres. Revista Decimonónica, 15 (1), 50-66. Recuperado de http://www.decimononica.org/solarte-15-1/.

Tauzin, I. (1995). La Narrativa Femenina en el Perú antes de la guerra del Pacífico. Revista Crítica Literaria Latinoamericana, XXI (42), 161-187.

Van Deusen, N. E. (2002). Voces y silencios: el género en la historia peruana (1977-2002). Revista Histórica, XXVI (1-2),125-186.

Villavicencio, M. (1992). Del silencio a la palabra. Mujeres peruanas en el siglo XIX-XX. Lima, Perú: Editorial del Centro de la mujer peruana Flora Tristán.

Villavicencio, M. (2013). Acción de las mujeres peruanas durante la guerra con Chile. Revista Debates en sociología, (10), 147-158.

Zegarra, M. (2011). María Jesús Alvarado Rivera: la construcción de una intelectual feminista en Lima, 1878-1915. Lima, Perú: UNMSM. 